This is an electronic reprint of the original article. This reprint may differ from the original in pagination and typographic detail.

Author(s): Nečas, Jindrich; Lehtonen, Ari; Neittaanmäki, Pekka

Title: $\quad$ On the Construction of Lusternik-Schnirelmann Critical Values with Application to Bifurcation Problems

Year: $\quad 1987$

Version:

Please cite the original version:

Nečas, J., Lehtonen, A. \& Neittaanmäki, P. (1987). On the Construction of LusternikSchnirelmann Critical Values with Application to Bifurcation Problems. Applicable Analysis, 25 (4), 253-268. doi:10.1080/00036818708839689

All material supplied via JYX is protected by copyright and other intellectual property rights, and duplication or sale of all or part of any of the repository collections is not permitted, except that material may be duplicated by you for your research use or educational purposes in electronic or print form. You must obtain permission for any other use. Electronic or print copies may not be offered, whether for sale or otherwise to anyone who is not an authorised user. 


\title{
On the Construction of Lusternik-Schnirelmann Critical Values with Application to Bifurcation Problems
}

\author{
Communicated by Gary F. Roach
}

\section{JINDRICH NEČAS}

Charles University, Faculty of Mathematics and Physics, KAM MFF UK Malostranske 2/25, CS-11800 Prague, Czechoslovakia

ARI LEHTONEN and PEKKA NEITTAANMÄKI

University of Jyväskylä, Department of Mathematics, Seminaarinkatu 15, SF-40100 Jyväskylä, Finland

AMS(MOS): 58E05, 65L15, 34B15, 47H15

Abstract An iterative method to construct Lusternik-Schnirelmann critical values is presented. Examples of its use to obtain numerical solutions to nonlinear eigenvalue problems and their bifurcation branches are given.

(Received for Publication 24 Fehruary 1987)

\section{Introduction}

Existence theorems for nonlinear eigenvalue problems of the form

$$
g^{\prime}(x)=\mu x
$$

where $g$ is a functional on a Hilbert space $H$ and $g^{\prime}(x)$ the corresponding gradient, are considered in many papers. The existence theorems are based on $t$ lsc existence of a critical vector with respect to the manifold $S=\{x \in H$ : 
$\|x\|=1\}$. Very general results on nonlinear eigenvalue problems in Banach spaces can be found in H. Amann ${ }^{1}$, and in E. Zeidler ${ }^{11}$, which also contains an extensive bibliography on critical point theories.

Iterative methods for the construction of all Lusternik-Schnirelmann critical values and critical vectors have been presented by J. Nečas ${ }^{7}$ and by A. Kratochvíl and J. Nečas ${ }^{4-5}$.

In this paper we shall give an extension of the method used in ${ }^{5}$ to study the eigenvalue problem (1.1). Also, we give examples of problems that have been tested numerically.

\section{Iterative construction of the first}

\section{Lusternik-Schnirelmann critical value}

Let $H$ be a real Hilbert space with inner product $(\cdot \mid \cdot)$ and norm $\|\cdot\|$. Furthermore, we set $S=\{x \in H:\|x\|=1\}$. Let $g$ be a continuously differentiable functional on $H$ such that the derivative $g^{\prime}$ is strongly continuous, i.e. for each sequence $\left(x_{n}\right)_{n=1}^{\infty} \subset H$ converging weakly to $x_{0} \in H$, the sequence $\left(g^{\prime}\left(x_{n}\right)\right)_{n=1}^{\infty}$ converges to $g^{\prime}\left(x_{0}\right)$.

Assume that the following conditions are fulfilled:

$$
\begin{gathered}
g(0)=0, \quad g^{\prime}(0)=0 ; \\
\text { if } g(x) \neq 0 \text { and }\|x\| \leq 1 \text { then } g^{\prime}(x) \neq 0 ;
\end{gathered}
$$

there exists a constant $c>0$ such that

$$
\left(g^{\prime}(x)-g^{\prime}(y) \mid x-y\right) \geq-c\|x-y\|^{2}
$$

holds for all $x, y \in H,\|x\| \leq 1,\|y\| \leq 1$.

For a given $x \in S$ we divide $g^{\prime}(x)$ into tangential and normal components, i.e. $g^{\prime}(x)=T g^{\prime}(x)+N g^{\prime}(x)$, where

$$
T g^{\prime}(x)=g^{\prime}(x)-\frac{\left(g^{\prime}(x) \mid x\right)}{\|x\|^{2}} x .
$$

Theorem 2.1. Let the above hypothesis be satisfied. Assume that $x_{1} \in S$ and $g\left(x_{1}\right)>0$. Let $0<\theta<1 / c$. Define a sequence $\left(x_{n}\right)_{n=1}^{\infty}$ by

$$
x_{n+1}=\frac{x_{n}+\theta g^{\prime}\left(x_{n}\right)}{\left\|x_{n}+\theta g^{\prime}\left(x_{n}\right)\right\|} \text {. }
$$


Then there exists a subsequence of $\left(x_{n}\right)_{n=1}^{\infty}$ converging to a solution of (1.1). Hence, there exists a point $x_{0} \in S$ and a number $\mu \in \mathbf{R}$ such that

$$
g^{\prime}\left(x_{0}\right)=\mu x_{0}
$$

Proof. First, note that $x \perp T g^{\prime}(x)$ for all $x \in S$. Put $\mu_{n}=\left(g^{\prime}\left(x_{n}\right) \mid x_{n}\right)$ and $r_{n}=\left\|x_{n}+\theta g^{\prime}\left(x_{n}\right)\right\|$. Then $r_{n}^{2}=\left\|\left(1+\theta \mu_{n}\right) x_{n}+\theta T g^{\prime}\left(x_{n}\right)\right\|^{2}=\left(1+\theta \mu_{n}\right)^{2}+$ $\theta^{2}\left\|T g^{\prime}\left(x_{n}\right)\right\|^{2}$.

From (2.3) we get $\mu_{n} \geq-c$. Since $0<\theta<1 / c$ we have $r_{n} \geq 1-\theta c>0$. Furthermore, from (2.5) we obtain the following identity

$$
\begin{aligned}
\left(g^{\prime}\left(x_{n}\right) \mid x_{n+1}-x_{n}\right) & =\frac{1}{\theta}\left(r_{n} x_{n+1}-x_{n} \mid x_{n+1}-x_{n}\right) \\
& =\frac{1}{\theta}\left(r_{n}-\left(x_{n} \mid x_{n+1}\right)-r_{n}\left(x_{n+1} \mid x_{n}\right)+1\right) \\
& =\frac{1}{2} \bar{\theta}\left(1+r_{n}\right)\left\|x_{n+1}-x_{n}\right\|^{2} .
\end{aligned}
$$

Next, we shall show that the sequence $\left(g\left(x_{n}\right)\right)_{n=1}^{\infty}$ is increasing. For this we note that using (2.3) we obtain

$$
\begin{aligned}
g(y)-g(x) & =\int_{0}^{1}\left(g^{\prime}(x+t(y-x)) \mid y-x\right) d t \\
& =\int_{0}^{1}\left(g^{\prime}(x+t(y-x))-g^{\prime}(x) \mid y-x\right) d t+\left(g^{\prime}(x) \mid y-x\right) \\
& \geq-\frac{c}{2}\|y-x\|^{2}+\left(g^{\prime}(x) \mid y-x\right) .
\end{aligned}
$$

Using this and (2.7) we get

$$
g\left(x_{n+1}\right)-g\left(x_{n}\right) \geq(1 / \theta-c)\left\|x_{n+1}-x_{n}\right\|^{2} .
$$

Hence the sequence $\left(g\left(x_{n}\right)\right)_{n=1}^{\infty}$ is increasing. Because of the strong continuity of $g^{\prime}$ the functional $g$ is bounded on $S$. Therefore, the limit $\lim _{n \rightarrow \infty} g\left(x_{n}\right)$ exists and

$$
\lim _{n \rightarrow \infty}\left\|x_{n+1}-x_{n}\right\|=0
$$


Since the sequence $\left(x_{n}\right)_{n=1}^{\infty}$ is bounded, it has a subsequence (we use the same notation for each subsequence) converging weakly to some $x_{0} \in H$. The strong continuity of $g^{\prime}$ yields $r_{n}^{2}=1+2 \theta\left(g^{\prime}\left(x_{n}\right) \mid x_{n}\right)+\theta^{2}\left\|g^{\prime}\left(x_{n}\right)\right\|^{2} \rightarrow$ $1+2 \theta\left(g^{\prime}\left(x_{0}\right) \mid x_{0}\right)+\theta^{2}\left\|g^{\prime}\left(x_{0}\right)\right\|^{2}=: r_{0}$.

Since $x_{n+1}=1 / r_{n}\left(x_{n}+\theta g^{\prime}\left(x_{n}\right)\right)$ we get $\left(r_{0}-1\right) x_{0}=\theta g^{\prime}\left(x_{0}\right)$. Furthermore, as $g\left(x_{0}\right) \geq g\left(x_{n}\right)>0$, we obtain from (2.1) and (2.2), $g^{\prime}\left(x_{0}\right) \neq 0$. Therefore $r_{0} \neq 1$. To show that the sequence $\left(x_{n}\right)_{n=1}^{\infty}$ converges to $x_{0}$ we write $(2.5)$ as

$$
\left(1-r_{n}\right) x_{n}=r_{n}\left(x_{n+1}-x_{n}\right)-\theta g^{\prime}\left(x_{n}\right) .
$$

Here the right hand side converges in norm to $-\theta g^{\prime}\left(x_{0}\right)$ and the left hand side to $1-r_{0}$. Since $1-r_{0} \neq 0$, we obtain the desired convergence from (2.9).

Corollary 2.2. Assume that instead of (2.3) the inequality

$$
\left(g^{\prime}(x)-g^{\prime}(y) \mid x-y\right) \leq c\|x-y\|^{2}
$$

holds for some $c>0$ and for all $x, y \in H,\|x\| \leq 1,\|y\| \leq 1$, and that $x_{1} \in S$ is such that $g\left(x_{1}\right)<0$. Let $-1 / c<\theta<0$. Define the sequence $\left(x_{n}\right)_{n=1}^{\infty}$ by (2.5). Then there exists a subsequence of $\left(x_{n}\right)_{n=1}^{\infty}$ converging to a solution of (2.6).

Proof. We can apply Theorem 2.1 to $-g$ and $-\theta$. The sequence $\left(g\left(x_{n}\right)\right)_{n=1}^{\infty}$ is now decreasing.

\section{Higher order critical points}

To study higher order critical points we recall some definitions concerning the Lusternik-Schnirelmann theory in an infinite dimensional Hilbert space.

We use the notion of the order of a set rather than the category or genus $^{2}$. Let $K$ be a symmetric closed set in $H$. We say that ord $K=0$ if $K$ is empty; that ord $K=1$ if $K=K_{1} \cup K_{2}$, where the $K_{i}$ are closed subsets of $K$ and neither $K_{1}$ nor $K_{2}$ contains antipodal points. In general, ord $K=n$ if 
$K=\cup_{i=1}^{n+1} K_{i}$, where the $K_{i}$ are closed subsets of $K$ not containing antipodal points and $n$ is the least possible number. Finally, ord $K=\infty$ if no such $n$ exists.

Let $V_{k}^{ \pm}$denote the set of all symmetric compact subsets $K$ of $S$ such that ord $K \geq k$ and $\pm g(x)>0$ on $K$. Denote

$$
\pm \gamma_{k}^{ \pm}=\left\{\begin{array}{l}
\sup _{\kappa \in V_{k}^{ \pm}} \min _{x \in K} \pm g(x), \text { if } V_{k}^{ \pm} \neq \emptyset, \\
0, \text { if } V_{k}^{ \pm}=\emptyset .
\end{array}\right.
$$

The fundamental theorem of the Lusternik-Schnirelmann theory states that there exists a sequence of critical points $x_{k}^{ \pm}$of $g$ such that $g\left(x_{k}^{ \pm}\right)=\gamma_{k}^{ \pm}$, $\pm \gamma_{k}^{ \pm} \searrow 0$ and $x_{k}^{ \pm} \rightarrow 0$ weakly. For a proof $\operatorname{see}^{1}$ or $^{11}$, Ch. 44 . In case $g(x)>0$ on $H$, a proof has been given in ${ }^{7}$. There the critical points are found as limits $\lim _{t \rightarrow \infty} x(t)$, where $x(t)$ is the solution of the differential equation on $S$

$$
\dot{x}=T g^{\prime}(x), \quad x(0)=x_{0} \in S .
$$

Our method using a discrete iteration seems to be more stable with respect to variations in $x_{0}$ than this one.

Let the assumptions of Theorem 2.1 hold for a functional $g$. Furthermore, assume that $g$ is even on $S$, i.e. $g(-x)=g(x)$ when $\|x\|=1$. We assume that $g$ has positive critical values. Let $\gamma_{1}^{+}$and $\gamma_{2}^{+}$be the first and second Lusternik-Schnirelmann critical values of $\left.g\right|_{S}, \gamma_{1}^{+}>\gamma_{2}^{+}$. Let there exist a positive constant $\varepsilon$ such that there are no critical values in the interval $] \gamma_{2}^{+}-\varepsilon, \gamma_{2}^{+}$[. Let $K_{1}$ be a compact symmetric subset of $S$ such that ord $K_{1} \geq 2, g(x)>0$ on $K_{1}$ and

$$
\gamma_{2}^{+}-\varepsilon<\min _{x \in K_{1}} g(x)<\gamma_{2}^{+} .
$$

We denote by $\varphi$ the function used to define the iteration in (2.5), i.e.

$$
\varphi(x)=\frac{x+\theta g^{\prime}(x)}{\left\|x+\theta g^{\prime}(x)\right\|} .
$$

Then $\varphi$ is a well-defined, odd continuous map $S \rightarrow S$. Choose $x_{1} \in K_{1}$, and put $x_{n+1}=\varphi\left(x_{n}\right)=\varphi^{n}\left(x_{1}\right)$, where $\varphi^{n}$ denotes the $n$-fold composition $\varphi \circ \cdots \circ \varphi$. 
Let $x_{n}^{(0)}$ be a vector from $K_{1}$ such that

$$
\min _{x \in K_{1}} g\left(\varphi^{n}(x)\right)=g\left(\varphi^{n}\left(x_{n}^{(0)}\right)\right)
$$

for any integer $n$.

Theorem 3.1. Let the above assumptions be fulfilled. Then the following assertions hold:

(i) $\lim _{n \rightarrow \infty} g\left(\varphi^{n}\left(x_{n}^{(0)}\right)\right)=\gamma_{2}^{+}$;

(ii) there exists $x^{(0)} \in K_{1}$ such that

$$
\lim _{n \rightarrow \infty} g\left(\varphi^{n}\left(x^{(0)}\right)\right)=\gamma_{2}^{+}
$$

(iii) there exists a subsequence of $\left(x_{n}^{(0)}\right)_{n=1}^{\infty}$ converging to $x^{(0)}$

(iv) for each $x^{(0)}$ satisfying condition (ii) there exists a subsequence of $\left(\varphi^{n}\left(x^{(0)}\right)\right)_{n=1}^{\infty}$ converging to some $x_{0}$ such that

$$
g^{\prime}\left(x_{0}\right)=\mu x_{0}
$$

Proof. From the proof of Theorem 2.1 (see (2.8)) we have

$$
g\left(\varphi^{n}(x)\right) \leq g\left(\varphi^{n+1}(x)\right)
$$

for each $x \in K_{1}$. Since $\varphi$ is an odd continuous operator from $S$ to $S$ we have

$$
\operatorname{ord} \varphi^{n}\left(K_{1}\right) \geq \operatorname{ord} K_{1} \geq 2
$$

From this, the definition of $\gamma_{2}^{+}$and (3.5) we get

$$
\lim _{n \rightarrow \infty} \min _{x \in K_{1}} g\left(\varphi^{n}(x)\right) \leq \gamma_{2}^{+}
$$

Let us denote

$$
\kappa=\lim _{n \rightarrow \infty} \min _{x \in K_{1}} g\left(\varphi^{n}(x)\right)=\lim _{n \rightarrow \infty} g\left(\varphi^{n}\left(x_{n}^{(0)}\right)\right) .
$$


By compactness there exists a subsequence of $\left(x_{n}^{(0)}\right)_{n=1}^{\infty}$ converging to $x^{(0)} \in K_{1}$.

According to Thcorem 2.1,

$$
\lim _{n \rightarrow \infty} g\left(\varphi^{n}\left(x^{(0)}\right)\right)
$$

is a critical value of $g$. Since there are no critical values in the interval ]$\gamma_{2}^{+}-\epsilon, \gamma_{2}^{+}[$, we obtain

$$
\lim _{n \rightarrow \infty} g\left(\varphi^{n}\left(x^{(0)}\right)\right) \geq \gamma_{2}^{+}
$$

Take $\eta>0$. Then by the definition of $x_{n}^{(0)}$ and the convergence $x_{n}^{(0)} \rightarrow x^{(0)}$ there exist numbers $n_{0}$ and $n_{1}$ such that

$$
g\left(\varphi^{n_{0}}\left(x_{n}^{(0)}\right)\right) \geq \gamma_{2}^{+}-\eta,
$$

for any integer $n \geq n_{1}$.

According to (3.5) and (3.8)

$$
g\left(\varphi^{n}\left(x_{n}^{(0)}\right)\right) \geq g\left(\varphi^{n_{0}}\left(x_{n}^{(0)}\right)\right) \geq \gamma_{2}^{+}-\eta,
$$

for any $n \geq \max \left\{n_{0}, n_{1}\right\}$ large enough. Therefore

$$
\kappa=\lim _{n \rightarrow \infty} g\left(\varphi^{n}\left(x_{n}^{(0)}\right)\right) \geq \gamma_{2}^{+}-\eta,
$$

and thus (i) follows.

The rest of the proof follows as in Theorem 2.1.

In a similar way we obtain the following result

Corollary 3.2. Let the assumptions of Theorem 9.1 be fulfilled. Let

$$
\gamma_{1}^{+} \geq \cdots \geq \gamma_{k}^{+}>\gamma_{k+1}^{+}=\cdots=\gamma_{k+l}^{+}>\gamma_{k+l+1}^{+}
$$

be the positive Lusternik-Schnirelmann values of $g \mid s$.

Let there exist a constant $\varepsilon>0$ such that there are no critical values in the interval $] \gamma_{k+l}^{+}-\varepsilon, \gamma_{k+l}^{+}[$. 
Let $K_{1}$ be a compact symmetric subset of $S$ such that

$$
\begin{gathered}
\operatorname{ord} K_{1} \geq k+1 \\
\gamma_{k+1}^{+}-\varepsilon<\min _{x \in K_{1}} g(x)<\gamma_{k+1}^{+} .
\end{gathered}
$$

For $x \in K_{1}$ let the sequence $\left(x_{n}^{(0)}\right)_{n=1}^{\infty}$ be defined by (9.4).

Then

$$
\lim _{n \rightarrow \infty} g\left(\varphi^{n}\left(x_{n}^{(0)}\right)\right)=\gamma_{k+l}^{+},
$$

and there exists a point $x^{(0)} \in K_{1}$ such that

$$
\lim _{n \rightarrow \infty} g\left(\varphi^{n}\left(x^{(0)}\right)\right)=\gamma_{k+l}^{+}
$$

Moreover, the assertions (iii) and (iv) of Theorem 9.1 hold.

Remark 9.9. Assume that instead of (2.3) the condition (2.10) holds and that $\theta$ is chosen as in Corollary 2.2. Then, with obvious modifications in Theorem 3.1 and Corollary 3.2 we can also construct negative critical values and corresponding critical points.

\section{Bifurcation of solutions}

We will use the previous results to find bifurcation branches for the equation (1.1). Let $g: H \rightarrow \mathbf{R}$ be a given function and for $r>0$ define $g_{r}: H \rightarrow$ $\mathbf{R}, g_{r}(x)=g(r x)$. Then $g_{r}^{\prime}(x)=\mu x$ if and only if $g^{\prime}\left(x_{r}\right)=\mu_{r} x_{r}$, where $x_{r}=$ $r x$ and $\mu_{r}=\mu r^{-2}$. It is immediately verified that if the second derivative $g^{\prime \prime}(0)$ exists and $\left(\mu_{0}, 0\right)$ is a bifurcation point of $(1.1)$, then $\mu_{0}$ is an eigenvalue of $g^{\prime \prime}(0)$.

To calculate solutions for the equation (1.1) in Section 6 we will use the following method: Let $\mu_{0} \neq 0$ be an eigenvalue and $x_{1}$ an eigenvector of $g^{\prime \prime}(0)$. Using Theorems 2.1 and 3.1 and Corollary 2.2 solve the equation $g_{r}^{\prime}(x)=\mu x, x \in S$. Then $\left(\mu_{r}, x_{r}\right)$ gives a bifurcation branch of (1.1).

In order to verify that this method can be applied we need the following result due to Krasnoselskii: 
Theorem 4.1. Let $B: H \rightarrow H$ be a linear, continuous and synmetric operator with $\mathcal{R}(B)$ closed and $0<\operatorname{dim} \mathcal{N}(B)<\infty$. Let $N$ be a continuously differentiable potential operator on a neighbourhood of the origin such that $\|N(x)\| /\|x\| \rightarrow 0$ as $x \rightarrow 0$. Then the point $(0,0)$ is a bifurcation point of the equation

$$
B x+N(x)=\epsilon x .
$$

A proof of this result can be found in ${ }^{11}$, Ch. 45; see also ${ }^{2}$, App. 1.

Now we can prove

Theorem 4.2. Let $g: H \rightarrow \mathbf{R}$ be a twice continuously differentiable function on a neighbourhood of the origin. Assume that $g^{\prime}$ is strongly continuous and that $g^{\prime \prime}(0)$ has an eigenvalue $\mu_{0} \neq 0$. Then the point $\left(\mu_{0}, 0\right)$ is a bifurcation point of (1.1).

Proof. We can write the equation (1.1) in the form

$$
\mu_{0} x-g^{\prime \prime}(0) x-\left(g^{\prime}(x)-g^{\prime \prime}(0) x\right)=\left(\mu_{0}-\mu\right) x .
$$

Denote $\varepsilon=\mu_{0}-\mu, B=\mu_{0} 1-g^{\prime \prime}(0)$ and $N(x)=-g^{\prime}(x)+g^{\prime \prime}(0) x$. From the strong continuity of $g^{\prime}$ it follow's that $g^{\prime \prime}(0)$ is compact. Therefore $\mathcal{R}$. $(B)$ is closed and $0<\operatorname{dim} \mathcal{N}(B)<\infty, \mathrm{cf}^{10}{ }^{10}$, Ch. X.5. Since $g^{\prime \prime}$ is continuous, we have $\|N(x)\| /\|x\| \rightarrow 0$ as $x \rightarrow 0$. Hence the conditions of Theorem 4.1 are fulfilled and the result follows.

\section{Application to differential equations}

We will apply the previous results to the following two point boundary value problem

$$
\left\{\begin{array}{l}
-u^{\prime \prime}=\lambda G^{\prime}(u) \\
u(\boldsymbol{a})=u(b)=0
\end{array}\right.
$$

on a bounded interval $] a, b[\subset \mathbf{R}$, where $G: \mathbf{R} \rightarrow \mathbf{R}$ is a given continuously differentiable function and $G^{\prime}(u)$ denotes the function $t \mapsto G^{\prime}(u(t))$. For partial differential equations the situation is analogous and will not be handled in this connection. 
We use the inner product $(u \mid v)=\left(u^{\prime} \mid v^{\prime}\right)_{L^{2}(a, b)}$ in the Sobolev space $H=H_{0}^{1}(a, b)$. Problem (5.1) is then equivalent to finding $u \in H$ such that

$$
\left(G^{\prime}(u) \mid v\right)_{L^{2}(a, b)}=\mu(u \mid v) \quad \text { for all } v \in H,
$$

where $\mu=1 / \lambda$.

We define $g: H \rightarrow \mathbf{R}$ by

$$
g(u)=\int_{a}^{b} G(u(t)) d t .
$$

Then the derivative of $g$ is given by

$$
\left(g^{\prime}(u) \mid v\right)=\int_{a}^{b} G^{\prime}(u(t)) v(t) d t \quad \text { for all } v \in H .
$$

Since $H$ embeds compactly into the space of continuous functions on the interval $[a, b]$, the derivative $g^{\prime}$ is strongly continuous.

We assume that $G(0)=G^{\prime}(0)=0$. In order to fulfil the condition (2.2) we assume that $G^{\prime}(r)$ does not vanish on any interval. If we assume that $G$ admits a continuous second derivative, then $g^{\prime \prime}$ exists and is given by

$$
g^{\prime \prime}(u)(h, k)=\int_{a}^{b} G^{\prime \prime}(u(t)) h(t) k(t) d t .
$$

It is easily verified that the condition (2.3) holds for the constant $c=$ $((b-a) / 2)^{2} \inf \left\{G^{\prime \prime}(r):|r| \leq \sqrt{(b-a) / 2}\right\}$, if $c<0$, and $c=0$ else.

Therefore, the linear eigenvalue problem $g^{\prime \prime}(0) u=\mu_{0} u$ is equivalent to the boundary value problem

$$
\left\{\begin{array}{l}
-\mu_{0} u^{\prime \prime}=G^{\prime \prime}(0) u \\
u(a)=u(b)=0
\end{array}\right.
$$

If $G^{\prime \prime}(0) \neq 0$ then the problem (5.3) has nonzero eigenvalues and we can apply Theorem 4.2 to prove that they give bifurcation branches for the problem (5.1). 


\section{Numerical examples}

We shall consider three examples where the method presented above is applied in connection with the finite element method. The problem (5.1) is solved by FEM with piecewise linear elements. We divide the interval $[a, b]$ into $n(h)$ subintervals $\left[a_{i}, a_{i+1}\right], h=a_{i+1}-a_{i}$. Let $\left(Q_{1}, \ldots, Q_{n(h)-1}\right)^{T}$ be the vector of the nodal values of the finite element solution. Then the finite element analogue of the problem $(5.1)$ reads

$$
A Q=\lambda G^{\prime}(Q)
$$

where $A$ is the stiffness matrix

$$
A=\frac{1}{h}\left(\begin{array}{cccccc}
2 & -1 & 0 & \ldots & 0 & 0 \\
-1 & 2 & -1 & \ldots & 0 & 0 \\
\vdots & \vdots & \vdots & \ddots & \vdots & \vdots \\
0 & 0 & 0 & \ldots & 2 & -1 \\
0 & 0 & 0 & \ldots & -1 & 2
\end{array}\right)
$$

and $G^{\prime}(Q)=h\left(G^{\prime}\left(Q_{1}\right), \ldots, G^{\prime}\left(Q_{n(h)-1}\right)\right)^{T}$.

The norms appeared above are computed here in the discrete form

$$
\|Q\|=\sqrt{(Q \mid A Q)} .
$$

The authors are indebted to Mr. T. Männikkö for his assistance in the following numerical tests.

Example 6.1. Consider the nonlinear eigenvalue problem

$$
\left\{\begin{array}{l}
\left.-u^{\prime \prime}(t)=\lambda \sin u(t), \quad t \in\right] 0,1[, \\
u(0)=u(1)=0 .
\end{array}\right.
$$

The simple eigenvalues of the linearized problem for (6.1) are $\lambda_{i}=i^{2} \pi^{2}$ and the corresponding eigensolutions are $\phi_{i}(t)=\sin \pi i t$. Consequently, the symmetric bifurcation points are $\left(\lambda_{i}, 0\right)$. In Figures $1-3$ we see the numerical results obtained with discretization parameter $h=1 / 32$ for the first, second and third bifurcation branches. On the left, $\max _{t}|u(t, \lambda)|$ is plotted as a function of $\lambda$, and on the right $u$ as a function of $(t, \lambda)$. 

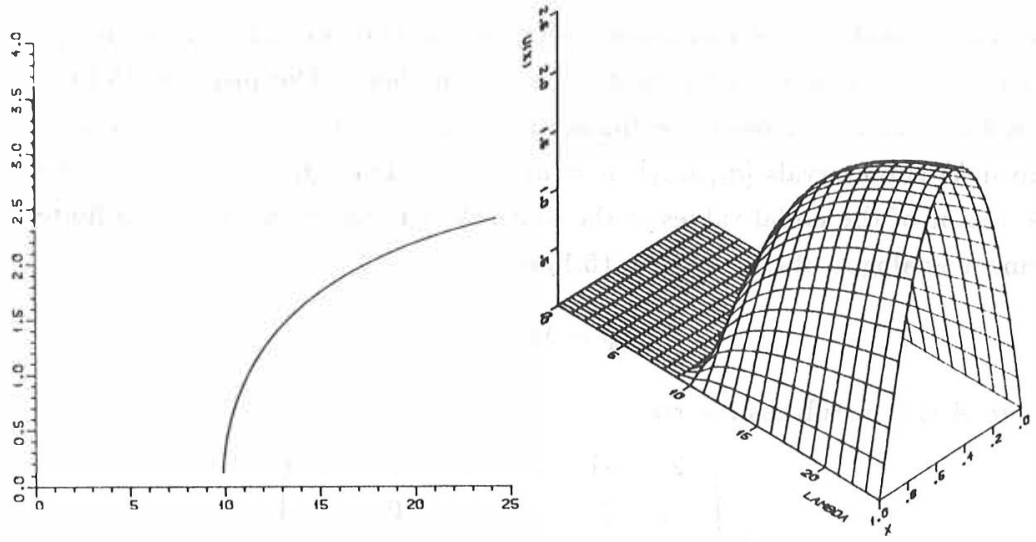

FIGURE 1 First bifurcation branch for $-u^{\prime \prime}=\lambda \sin u$.
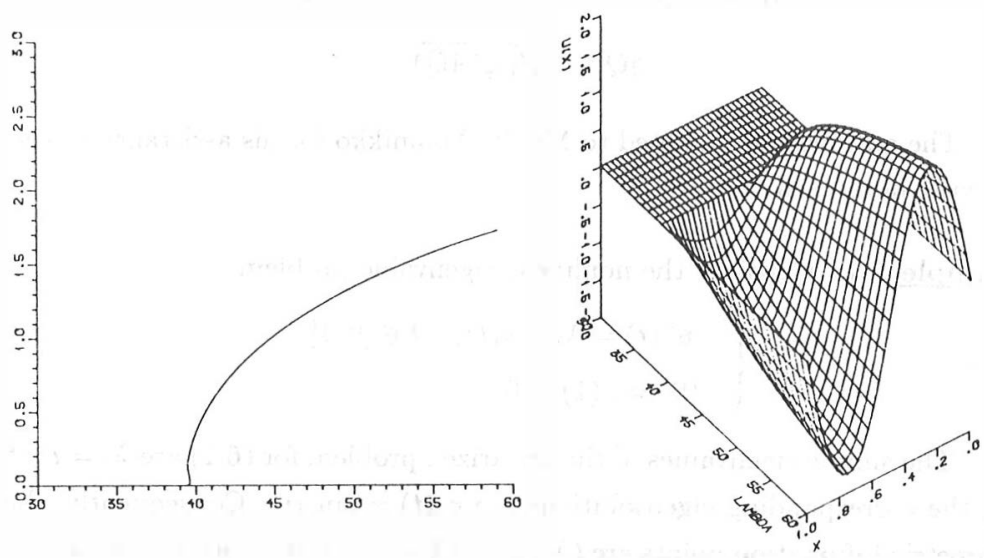

FIGURE 2 Second bifurcation branch for $-u^{\prime \prime}=\lambda \sin u$. 

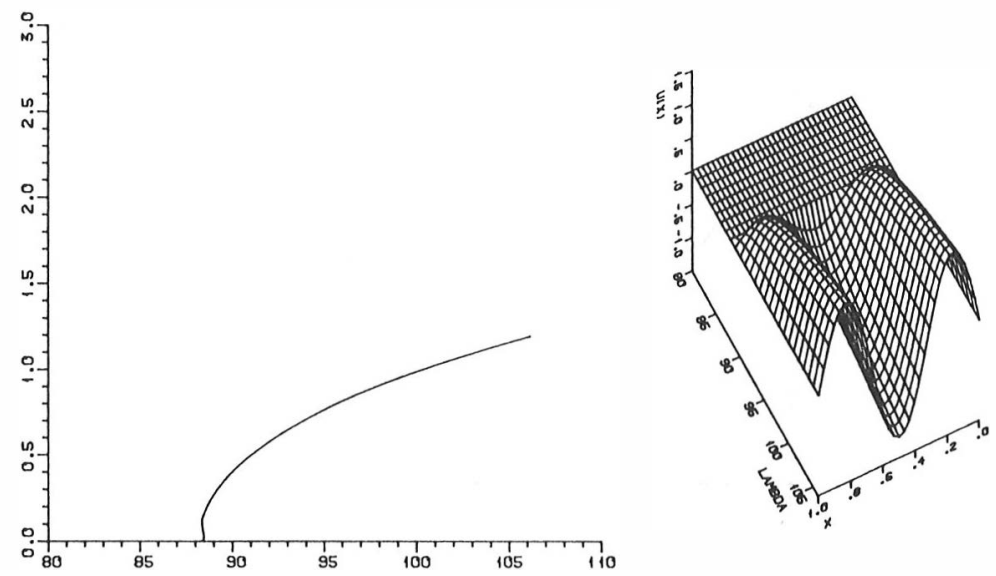

FIGURE 3 Third bifurcation branch for $-u^{\prime \prime}=\lambda \sin u$.

Example 6.2. Consider the nonlinear eigenvalue problem

$$
\left\{\begin{array}{l}
\left.-u^{\prime \prime}(t)=\lambda\left(u(t)(1-\sin u(t))+(u(t))^{3}\right), \quad t \in\right] 0,1[ \\
u(0)=u(1)=0
\end{array}\right.
$$

In Figure 4 we see the behaviour of $\max _{t \in] 0,1[}|u(t, \lambda)|$ for $\left.\lambda \in\right] 0,15[$, $h=1 / 32$. At the point $\lambda \approx 12.7$ a turning point occurs.

Example 6.3. Consider the nonlinear eigenvalue problem

$$
\left\{\begin{array}{l}
\left.-u^{\prime \prime}(t)=\exp (u(t) /(1+D u(t))), \quad t \in\right]-1,1[ \\
u(-1)=u(1)=0
\end{array}\right.
$$

where $D \geq 0$.

Figures 5-6 show the numerical results with $h=1 / 32$ for various parameters $D$. In Figure 5 the illustration of $\max _{t \in]-1,1[}|u(t, \lambda)|$ for $\left.\lambda \in\right] 0,2[$ with $D=0$ and $D=0.1$. Figure 6 shows the result with $D=0.2$ and $D=0.3$. 


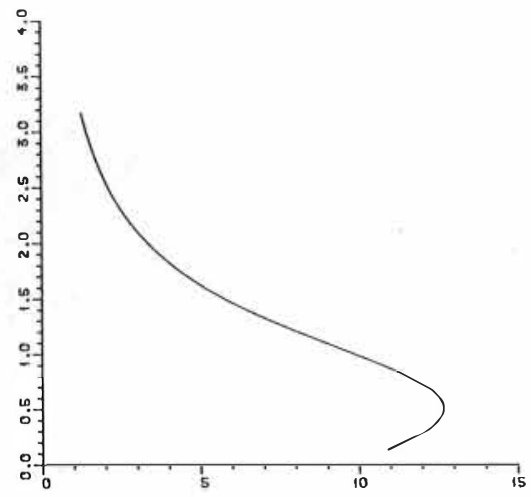

FIGURE 4 First bifurcation branch for $-u^{\prime \prime}=\lambda\left(u(1-\sin u)+u^{3}\right)$.
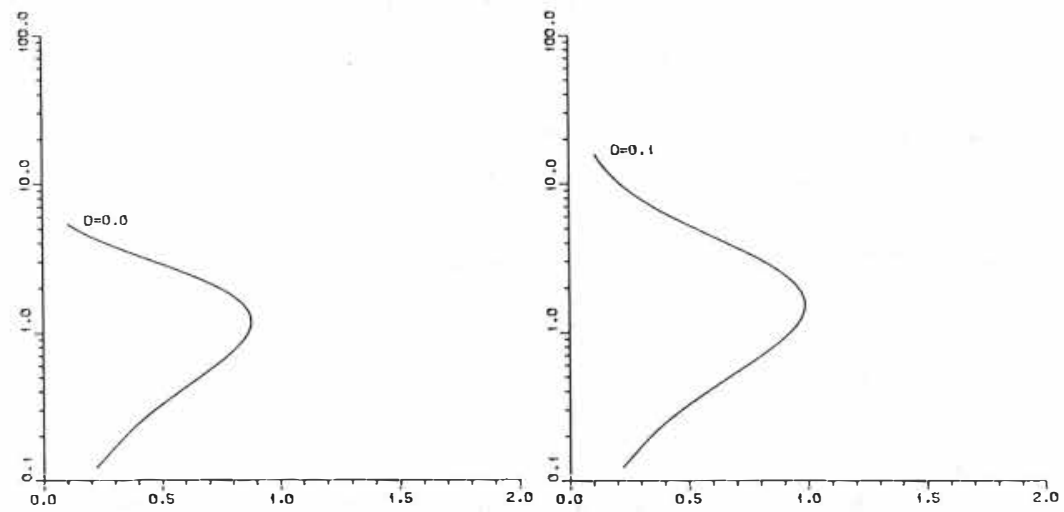

FIGURE 5 Bifurcation of $-u^{\prime \prime}=\exp (u /(1+D u))$, $D=0$ (left), $D=0.1$ (right). 

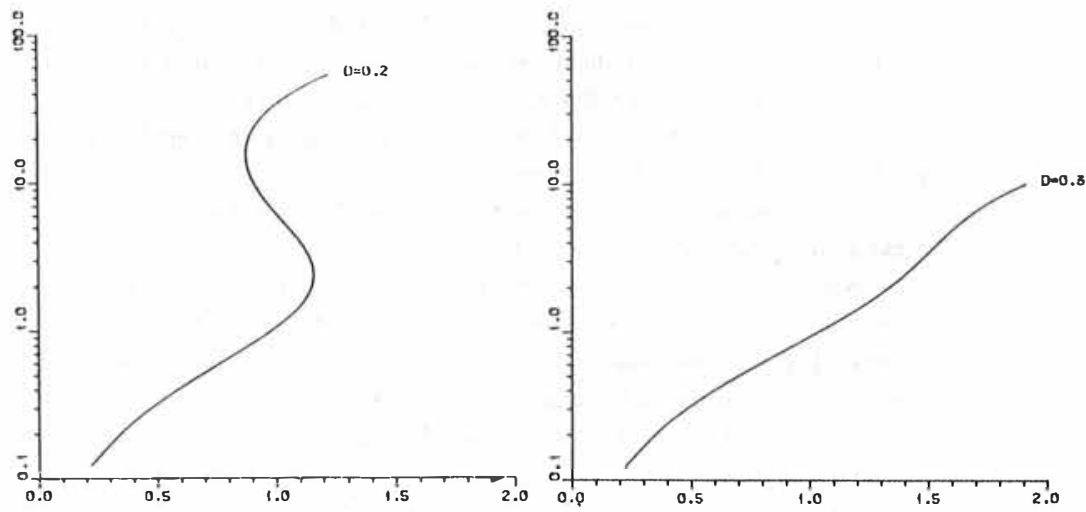

FIGURE 6 Bifurcation of $-u^{\prime \prime}=\exp (u /(1+D u))$, $D=0.2$ (left), $D=0.3$ (right).

As a summary of the above results we can say that by the method presented here we were able to find the bifurcation point or turning point with a relatively good accuracy even with a rough discretization parameter. Those examples are often used in the literature to test numerical methods for nonlinear problems. Our results agree well with those given in the literature, cf. ${ }^{3,6,8}$.

The method, of course, works for nonlinear eigenvalue problems for PDE's. These applications will be reported in a forthcoming paper.

\section{$\underline{\text { References }}$}

1. H. Amann: Lusternik-Schnirelmann Theory and Non-Linean Eigenvalue Problems. Math. Ann. 199, 55-72 (1972)

2. S. Fučik, J. Nečas, J. Souček and V. Souček: Spectral Analysis of Nonlinear Operators. Berlin-Heidelberg-New York. Lecture Notes in Mathematics 346, SpringerVerlag 1973

3. R. Glowinski, H. Keller and L. Reinhart: Continuation-conjugate gradient methods for least square solution of nonlinear boundary value problems. INRIA, Rappoits de Recherche 141 (1982) 
4. A. Kratochvil and J. Nećas : Secant Modulus Method for the Construction of a Solution of Nonlinear Eigenvalue Problems. Boll. Un. Mat. Ital. B(5) 16, 694-710 (1979)

5. A. Kratochvil and J. Nećas: Gradient Methods for the Construction of LusternikSchnirelmann Critical Values. RAIRO Anal. Numér. 14, 43-54 (1980)

6. M. D. Mittelman and H. Weber (ed.): Bifurcation Problems and Their Numerical Solution. Basel. ISNM 54, Birkhäuser 1980

7. J. Nečas: An Approximate Method of Finding Critical Points of Even Functionals. Proc. Steklov Inst. Math. 134, 267-272 (1975)

8. P. Neittaanmäki and $K$. Ruotsalainen: On the numerical solution of the bifurcation problem for the sine-Gordon equation. Arab. J. Math. (1986). To appear

9. L. Nirenberg: Topics in Nonlinear Functional Analysis. New York University. Lecture Note, Courant Institute of Mathematical Sciences 1974

10. K. Yosida : Functional Analysis. Fourth Edition. New York-Berlin-Heidelberg-Tokyo. Grundlehren der mathematischen Wissenschaften 123, Springer-Verlag 1974

11. E. Zeidler : Nonlinear Functional Analysis and its Applications III. Variational Methods and Optimization. New York-Berlin-Heidelberg-Tokyo. Springer-Verlag 1985 